\title{
uPAR-targeted Optical Imaging Contrasts as Theranostic Agents for Tumor Margin Detection
}

\author{
Lily Yang ${ }^{1,2}$, Hari Krishna Sajja ${ }^{1}$, Zehong Cao ${ }^{1}$, Weiping Qian ${ }^{1}$, Laura Bender ${ }^{3}$, Adam I. Marcus ${ }^{3}$, \\ Malgorzata Lipowska², William C. Wood ${ }^{1}$, and Y. Andrew Wang ${ }^{4}$ \\ 1. Department of Surgery, Emory University School of Medicine, 1365-C Clifton Road, Atlanta, GA, USA. \\ 2. Department of Radiology and Imaging Sciences, Emory University School of Medicine, 1364-C Clifton Road, Atlanta, GA, USA. \\ 3. Department of Hematology and Oncology, Emory University School of Medicine, 1365-C Clifton Road, Atlanta, GA, USA. \\ 4. Ocean Nanotech, LLC, Springdale, AR, USA.
}

$\triangle$ Corresponding author: Lily Yang, MD. PhD, Department of Surgery, Winship Cancer Institute, Emory University School of Medicine, Clinic C, C-4088, 1365-C Clifton Road, Atlanta, GA 30322. Phone: (404) 778-4269; Fax: (404) 778-5530; E-mail: lyang02@emory.edu

( ) Ivyspring International Publisher. This is an open-access article distributed under the terms of the Creative Commons License (http://creativecommons.org/ licenses/by-nc-nd/3.0/). Reproduction is permitted for personal, noncommercial use, provided that the article is in whole, unmodified, and properly cited.

Received: 2013.08.14; Accepted: 2013.10.15; Published: 2013.12.17

\begin{abstract}
Complete removal of tumors by surgery is the most important prognostic factor for cancer patients with the early stage cancers. The ability to identify invasive tumor edges of the primary tumor, locally invaded small tumor lesions, and drug resistant residual tumors following neoadjuvant therapy during surgery should significantly reduce the incidence of local tumor recurrence and improve survival of cancer patients. In this study, we report that urokinase plasminogen activator (UPA) and its receptor (UPAR) are the ligand/cell surface target pair for the development of targeted optical imaging probes for enhancing imaging contrasts in the tumor border. Recombinant peptides of the amino terminal fragment (ATF) of the receptor binding domain of uPA were labeled with near infrared fluorescence (NIR) dye molecules either as peptide-imaging or peptide-conjugated nanoparticle imaging probes. Systemic delivery of the uPAR-targeted imaging probes in mice bearing orthotopic human breast or pancreatic tumor xenografts or mouse mammary tumors led to the accumulation of the probes in the tumor and stromal cells, resulting in strong signals for optical imaging of tumors and identification of tumor margins. Histological analysis showed that a high level of uPAR-targeted nanoparticles was present in the tumor edge or active tumor stroma immediately adjacent to the tumor cells. Furthermore, following targeted therapy using uPAR-targeted theranostic nanoparticles, residual tumors were detectable by optical imaging through the imaging contrasts produced by NIR-dye-labeled theranostic nanoparticles in drug resistant tumor cells. Therefore, results of our study support the potential of the development of uPAR-targeted imaging and theranostic agents for image-guided surgery.
\end{abstract}

Key words: uPAR, optical imaging, theranostic nanoparticles, tumor margin, and image-guided surgery

\section{Introduction}

Surgery is the most effective treatment for cancer patients diagnosed with the early stage diseases. Typically, a surgeon removes the tumor along with surrounding non-cancerous tissues. The outside of the tumor, or tumor margin, is determined by pathological examination of the resected tissues during and post surgery. If the tumor border is more than $1 \mathrm{~mm}$ away from the resection edge of the tissue, this is considered to be a negative margin or R0 status [1-4]. However, detection of tumor cells at the edge of the resected tissue indicates a positive margin or R1 status $[1,2,5-7]$. Clinical studies have shown that the tumor margin status is a key prognostic factor for local tumor recurrence and survival of cancer patients for 
many types of resectable human cancers [5, 6, 8-10]. Cancer patients with a positive tumor margin often need additional surgery to remove the residual tumors, or post-operative chemo- or radiotherapy to prevent tumor recurrence $[7,11,12]$. Therefore, a curative surgical resection by achieving a negative tumor margin has been the most important goal for the surgical treatment of cancer patients.

At present, the most common clinical procedures for evaluating tumor margin status are intraoperative gross examination and frozen tissue section, and post-surgery histological analysis of resected tumor tissues $[1,5-7,13]$. It is clear that those intraoperative evaluations are not reliable since post-operative pathological analysis of the tumor tissues usually reveals high percentages of the tissues with positive tumor margins $[1,7,11,12]$. For example, 20 to $50 \%$ of breast patients after lumpectomy and $14 \%$ to $76 \%$ of pancreatic cancer patients after the Whipple procedure were found having positive tumor margins [4, $10,12,14]$. The major challenge in accurate identification of the tumor margin is that invasive tumor lesions don't usually have a well defined tumor boundary and sometimes small satellite tumors localize in the surrounding normal tissues $[1,2,4]$. The presence of fibrosis and inflammatory changes associated with preoperative chemotherapy or tumor-induced obstruction makes it extremely difficult for surgeons to determine the tumor margin [15 ]. Additionally, limited numbers of frozen tissue sections can be sampled around the tumor during surgery and the likelihood of underestimation of the tumor involvement in the margin is high $[1,6,7]$. Therefore, there is an urgent need to develop sensitive and accurate intraoperative imaging approaches for the detection of tumor margins.

Recent advances in the development of molecular imaging contrasts make it possible to visualize tumors using non-invasive as well as intraoperative imaging approaches [16-24]. Antibodies, peptides, and small molecules have been labeled with fluorescent dyes for generating targeted optical imaging probes [17-19, 25, 26]. Those targeting ligands can also be conjugated to various nanoparticles to produce nanoparticle imaging probes [20, 27-30]. Results of previous studies using targeted imaging probes showed the feasibility of the detection of tumors in animal tumor models and in humans. In a recent clinical trial, fluorescein isothiocyanate labeled folic acid was used for intraoperative optical imaging of ovarian cancers in the cancer patients [19].

The production of a targeted imaging contrast requires a pair of a cell surface target and a high affinity targeting ligand. An ideal cell surface molecule for developing targeted imaging agents should be overexpressed in tumor cells and has a high affinity ligand for recognition of the molecule that is used for directing nanoparticles. To increase specificity and sensitivity of optical imaging of tumor margins, it is also critical that the selected cell surface target should be highly expressed in the tumor boundary. Increasing evidence from histological analysis of human cancer tissues supports the potential of targeting urokinase plasminogen activator receptor (uPAR) for imaging tumor margins [31-35]. Urokinase plasminogen activator ( $\mathrm{uPA})$ is a serine protease that interacts with its receptor, uPAR, to regulate multiple pathways involved in matrix degradation, cell motility, metastasis, and angiogenesis [31]. In the majority of normal tissues or organs, the level of uPAR is very low or undetectable except for a low level of expression in macrophages, granulocytes, the uterus, thymus, kidney and spleen [36]. However, UPAR is highly expressed in many tumor types, such as breast, pancreatic, lung, and ovarian cancers [32, 35, 37]. Studies have demonstrated that a high level of uPAR expression in tumor cells correlates with aggressive tumor types, tumor metastasis, and poorer prognosis $[31,32,38]$. About 60 to $90 \%$ of invasive breast cancer tissues and $54 \%$ of the early stage of ductal carcinoma in situ (DCIS) expressed a high level of UPAR while normal breast tissues lack the expression [32, 39]. Although uPAR may be a potential target for all breast cancer subtypes, a recent study showed that triple negative breast cancer tissues expressed a higher level of uPAR compared to luminal breast cancer subtype [40]. Additionally, 86 to $90 \%$ of pancreatic cancer tissues had a high level of uPAR, but its expression was not found in the normal pancreas or pancreatic tissues with chronic pancreatitis [35, 41]. About $58 \%$ of pancreatic cancer tissues have uPAR gene amplification and the level of UPAR mRNA in pancreatic cancer tissues was 9.6 folds over adjacent normal tissues [41, 42]. Results from two studies of 15 or 29 pancreatic cancer biomarkers have placed UPAR as the top biomarker to distinguish between pancreatic cancer and normal pancreas or chronic pancreatitis $[41,42]$.

Unlike many other tumor cell surface biomarkers, uPAR is highly expressed in the tumor cells at the invasive edge and the tumor stroma, including angiogenic endothelial cells, active fibroblasts, and tumor associated macrophages [33, 38, 39, 43, 44]. Expression of UPAR in the tumor stroma is important for the detection of the satellite tumor lesions that are smaller than $1 \mathrm{~mm}^{3}$, which lack tumor angiogenesis and have a low efficiency in delivery of tumor cell-targeted imaging probes into the tumor. Therefore, those unique characteristics of UPAR make it a suitable cell target for the development of optical 
imaging probes for the detection of tumor margin.

In this study, we developed UPAR targeted optical imaging probes using amino terminal fragment (ATF) of uPA either as near infrared fluorescence (NIR) dye-labeled recombinant 135-amino acid peptides or NIR-dye-ATF peptides conjugated to polymer coated magnetic iron oxide nanoparticles (IONPs). Selective accumulation of the imaging probes in tumors, especially in the tumor margin, following systemic delivery of the targeted nanoparticles has been demonstrated in orthotopic breast and pancreatic cancer mouse models.

\section{Materials and Methods}

\section{Cell lines}

MDA-MB-231 human breast cancer cell line was purchased from American Type Culture Collection (ATCC, Rockville, MD). Luciferase gene stably transfected MIA PaCa-2 human pancreatic cell line was provided by Dr. Rosa Hwang, MD Anderson Cancer Center, Houston, TX). Both cell lines were cultured in DMEM medium containing $10 \%$ fetal bovine serum (FBS). The MCF-10 DCIS (or MCF-10DCIS.COM) human breast cancer cell line was obtained from Asterand US, Detroit, MI, and cultured in DMEM/F12 medium supplemented with $5 \%$ horse serum. $4 \mathrm{~T} 1$ mouse mammary tumor cell line was kindly provided by Dr. Fred R. Miller at Wayne State University, Detroit, MI. 4T1 cells were cultured in DMEM medium with $10 \%$ FBS.

\section{Animal tumor models}

The 4T1 mouse mammary tumor model was established by injecting $2 \times 10^{6}$ of $4 \mathrm{~T} 1$ cells into the mouse mammary fat pad in 6- to 8-week old female Balb/c or nude mice (Harlan Laboratories, Indianapolis, IN). 4T1 tumors grew to 5 to $10 \mathrm{~mm}$ in diameter measured using a caliper, in 10 to 14 days. $1 \times 10^{7}$ of human MDA-MB-231 cells were injected into the mammary fat pad of 6 to 8 weeks old female nude mice and the tumor xenograft reached the optimal imaging size of 5 to $10 \mathrm{~mm}$ in 3 to 4 weeks. To generate an orthotopic breast cancer model with both early stage ductal carcinoma in situ (DCIS) and invasive cancer characteristics, $5 \times 10^{6}$ of MCF-10DCIS cells were mixed with Matrigel (BD Biosciences, San Jose, CA) and then injected into the mammary fat pad of nude mice. MCF-10 DCIS tumors grew to 5 to $10 \mathrm{~mm}$ in diameter in 14 to 20 days.

The orthotopic human pancreatic cancer xenograft model was established using a surgical procedure. Under anesthesia, $5 \times 10^{6}$ of fire-fly luciferase gene stably transfected MIA PaCa- 2 cells were injected into the pancreas of 6 to 8 weeks old female nude mice. Pancreatic tumor xenografts reached 5 to 8 $\mathrm{mm}$ in diameter and were ready for experiments in about 3 to 4 weeks. The growth of orthotopic pancreatic cancer xenografts was monitored by bioluminescence imaging. All animal study protocols were approved by the Institute of Animal Use Committee of Emory University.

\section{Production of recombinant targeting ligands}

uPAR targeted mouse ATF peptides were produced from $\mathrm{pET101/D-TOPO}$ expression vector containing a cDNA fragment encoding amino acids 1 to 135 of mouse uPA $[27,34]$. Human ATF peptides were produced from a pET20a plasmid with the human ATF gene. Both mouse and human ATF peptides (17 $\mathrm{kDa})$ were produced in E. coli BL21 bacterial expression system and then purified from bacterial extracts under native conditions using a Ni2+NTA-agarose column (Qiagen, Valencia, CA).

Human single chain epidermal growth factor receptor (EGFR) antibody (ScFvEGFR) was produced in TG1 E. coli competent cells (Biochain Institute, Inc, Hayward, CA) using ScFv B10 plasmid [28]. Recombinant ScFvEGFR proteins $(25 \mathrm{kDa})$ were obtained from the bacterial lysates of $\mathrm{scFv}$ B10 transformed TG1 competent cells after Ni2+ NTA-agarose column separation under native conditions (Qiagen, Valencia, CA).

\section{Production of targeted optical imaging probes}

In this study, we produced five different optical imaging probes targeting to two cell surface receptors, UPAR and EGFR. These included uPAR-targeted Cy5.5-ATF (human or mouse), NIR-830-ATF-IONP, NIR-830-ATF-IONP-doxorubicin (Dox), and IRDye 800-ScFvEGFR (Figure 1)

Peptide-based probe: Three near infrared (NIR) dyes at a ratio of one targeting peptide to 4 dye molecules were used to label targeting ligands. Excitation and emission wavelengths of the NIR dye molecules are shown in Figure 1. Cy5.5 ${ }^{\mathrm{TM}}$ maleimide (GE Healthcare, Piscataway, NJ) was conjugated to reactive thiol group of the peptides using the manufacture's protocol. IRDye® $800 \mathrm{CW}$ NHS (LI-COR, Lincoln, NE) was labeled to active amine groups of the targeting peptides. A maleimide form of near infrared dye-830 (NIR-830 maleimide) was synthesized from IR-783 (Sigma-Aldrich, St Louis, MO) in our group and was conjugated to the thiol group of the targeting peptides based on the protocol developed in our laboratory (Figure 1) $[45,46]$. After 4 hours of the conjugation reaction, free dye molecules were separated from the dye-peptide conjugates using a Nanosep 3k OMEGA column (Pall Corp, Ann Arbor, MI). As a non-targeted control, mouse serum albumin (MSA) (Sigma-Aldrich) was labeled with NIR dye molecules 
using the method as described above.

uPAR-targeted optical imaging nanoparticle probes and theranostic nanoparticles: Magnetic iron oxide nanoparticles (IONPs, $10 \mathrm{~nm}$ core size) coated with amphiphilic polymers (Ocean Nanotech, LLC, Springdale, AR) were conjugated with NIR-dye 830-labeled ATF peptides at a ratio of 1 nanoparticle to 15 ATF peptides via cross-linking of carboxyl groups of the amphiphilic polymer to amino side groups of the peptides (Figure 1). The final nanoparticle conjugates were purified using Nanosep 100k column. A non-targeted control NIR-830-MSA-IONP was produced using the same protocol. To produce ATF-IONPs carrying a chemotherapy drug, doxorubicin HCI (Dox) (Polymed Therapeutics, Houston, TX) was dissolved in methanol and then added to ATF-IONPs at a ratio of $1 \mathrm{mg}$ Dox to $1 \mathrm{mg}$ of iron equivalent IONPs. After rotating at room temperature for $4 \mathrm{hrs}$, free Dox was separated from the encapsulated Dox using Nanosep 100k column filtration. Under high $\mathrm{pH}$ conditions, over $95 \%$ of Dox molecules were encapsulated into the hydrophobic space between the IONP core and amphiphilic polymer. The final hydrodynamic sizes of NIR-830-ATF-IONP and NIR-ATF-IONP-Dox were $\sim 20$ and $30 \mathrm{~nm}$, respectively, as determined using Zeta-sizer Nano (Malvern Instruments Inc., Southborough, MA).

\section{Histological analysis}

Following systemic delivery of the imaging probes and optical imaging procedure, the mice were sacrificed. Tumor and normal tissues were collected and then frozen in liquid nitrogen or fixed with $10 \%$ buffered formalin. $5 \mu \mathrm{m}$ of frozen or paraffin tissue sections were cut for histological analysis. H\&E
A.

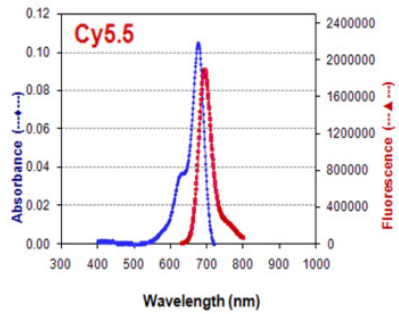

C.

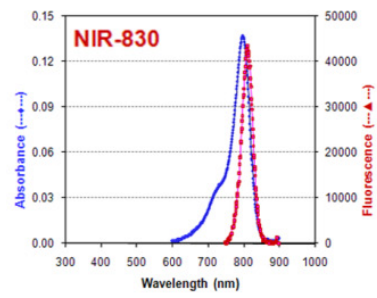

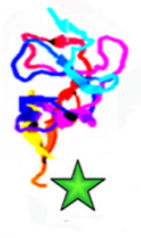

Cy5.5-ATF
B.
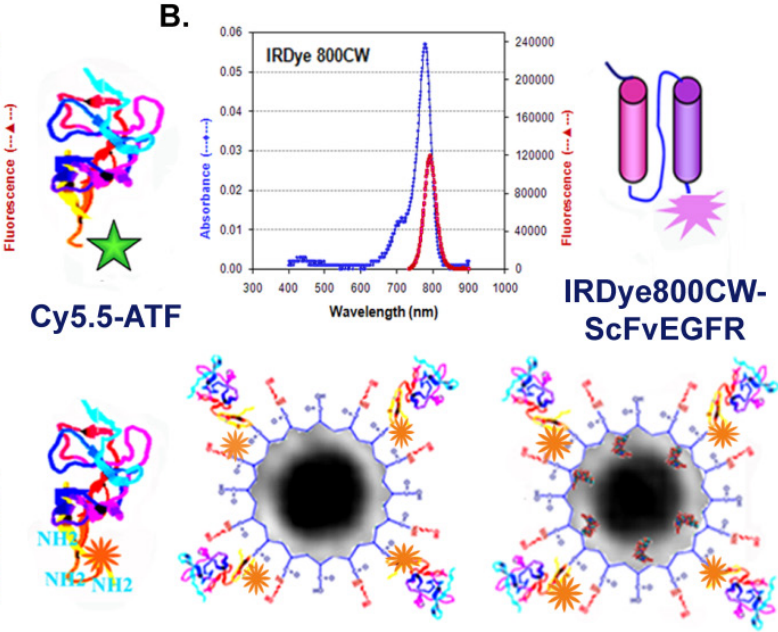

NIR-830-ATF

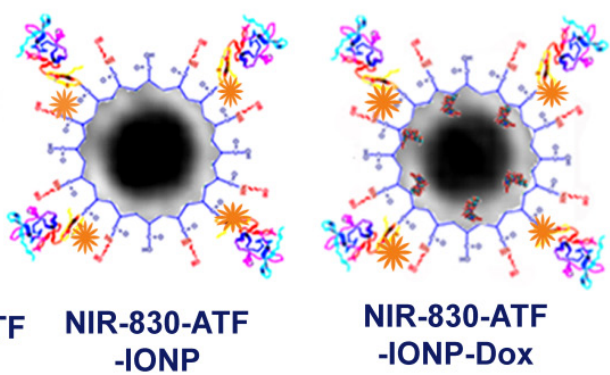

staining was conducted to determine morphological and pathological characteristics of tumors. Prussian blue staining was used to determine the accumulation of the iron nanoparticles in the tumor tissue sections using our established methods [23].

\section{Immunofluorescence labeling}

Frozen tissue sections of breast and pancreatic tumor xenografts were incubated with an anti-uPAR polyclonal rabbit antibody (Santa Cruz Biotechnology, Santa Cruz, CA), followed by Alexa Fluor 555-goat anti-rabbit IgG (Invitrogen, Carlsbad, CA). Mouse endothelial cells were identified using a FITC-labeled rat anti-mouse CD31 antibody (BD Biosciences, San Diego, CA). Mouse macrophages were identified using Alexa Fluor 488-labeled rat anti-mouse CD68 antibody (AbD Serotec, Raleigh, NC). Some tissue slides were doubly stained with uPAR and CD68, uPAR and CD31, or CD68 and Prussian blue staining. The tissue sections were labeled with UPAR antibody and then Alexa Fluor 555-goat anti-rabbit IgG using the method as described above. FITC-labeled rat anti-mouse CD31 antibody or Alexa Fluor 488-labeled rat anti-mouse CD68 antibody was then added to the tissue section for one hour. After washing with PBS, the tissue section was counter-stained with Hoechst 33342. For double labeling of CD68 and Prussian blue staining, tissue sections were incubated with Prussian blue staining solution for 2 hours. After washing thoroughly with PBS, Alexa Fluor 488-labeled rat anti-mouse CD68 antibody was added to the tissue section for 1 hour. Images were acquired at 20X magnification using a Zeiss Axioplan 2 upright fluorescence microscope.

Figure I. Schematic of optical imaging probes labeled with different NIR dyes. A. Cy5.5-recombinant ATF peptide imaging probe has an excitation wavelength of $680 \mathrm{~nm}$ and an emission wavelength of $694 \mathrm{~nm}$. B. IRDye800CW labeled single chain antibody (ScFvEGFR) imaging probe has an excitation wavelength of $780 \mathrm{~nm}$ and emission wavelength of $790 \mathrm{~nm}$. C. Three NIR-830 dye-labeled optical imaging probes were produced, including NIR-830-ATF peptide probe, NIR-830-ATF-IONP nanoparticle probe, and NIR-830-ATF- theranostic IONP carrying Dox. NIR-830 dye-labeled probes have an excitation wavelength of 800 nm and emission wavelength of $825 \mathrm{~nm}$. 


\section{NIR optical imaging}

For optical imaging of the mice that received Cy5.5 or IRDye 800 dye labeled imaging probes, the mice were placed on an alfalfa-free rodent diet (Harlan Teklad, Madison, WI) 3 to 5 days before the experiment to reduce body background. However, special diet was not required for the mice that received NIR-830 dye-labeled imaging probes.

Optical imaging was performed using either the OV-100 Small Animal imaging system (Olympus America Inc., Center Valley, PA) or Kodak FX in vivo imaging system (Carestream Health, Inc, New Haven, CT). For OV-100 imaging system, optical images were taken using 0.14 to $0.8 \mathrm{x}$ magnifications and the Xenon lamp. For Cy5.5 dye-labeled probes, a set of HQ595-635 nm (excitation) and Q675/50m (emission) was used to capture images. For IRDye 800 and NIR-830 dye labeled probes, a set of 730AF45 nm (excitation) and 770ALP (emission) was used to capture images. A bright field image was taken and overlaid with the optical image to show the location of the imaging signals.

All optical images taken by the Kodak FX in vivo imaging system were obtained from the mice that received systemic delivery of NIR-830-dye labeled IONP probes. Optical images were taken using an 800 $\mathrm{nm}$ excitation and $850 \mathrm{~nm}$ emission filter set with a 90-second exposure time and a Gamma value of 0.2. For each NIR image, a corresponding $\mathrm{X}$-ray image was taken to provide anatomic location of the tumor.

\section{Results}

\section{UPAR expression and targeted optical imaging in an orthotopic human breast cancer xeno- graft model}

It is well known that breast cancer is a highly heterogeneous disease with a wide range of pathological and phenotypic characteristics. DCIS of the breast is the early cancer stage with proliferating pre-invasive breast cancer cells inside the breast duct but have not breached the ductal basement membrane $[39,47]$. In this study, we used a human breast cancer xenograft model derived from the MCF-10DCIS cell line that contained a breast cancer progenitor cell population and gave rise to heterogeneous tumor lesions [47]. MCF-10 DCIS cells produce rapidly growing tumors in nude mice with predominant comedo DCIS lesions during the first three weeks of the tumor growth and gradually progressing to invasive tumors in 4 to 8 weeks (Figure 2A). Using an antibody against both human and mouse UPAR, we found that UPAR is highly expressed in the majority of DCIS nodules. However, the level of the expression varied among DCIS nodules with some expressing a very high level, while others have low to intermediate levels of UPAR (Figure 2). Furthermore, activated macrophages in the tumor stroma expressed a very high level of uPAR (Figure 2B). Even in some DCIS lesions with a low or absence of UPAR expression, the tumor stroma immediately adjacent to the tumor nodules still contained UPAR positive cells (Figure 2B). In invasive tumor lesions, uPAR is expressed in most tumor cells with a higher level in the tumor edge (Figure 2B).

The mice bearing MCF-10DCIS tumors received a tail vein injection of $50 \mu \mathrm{g}$ of NIR-830 dye labeled human ATF peptides. 24 hours after injection, optical imaging using the OV-100 in vivo imaging system showed a strong NIR imaging signal in an orthotopic DCIS lesion, which clearly delineated the tumor boundary (Figure $2 \mathrm{C}$ ).

\section{Comparison of optical signals in human breast cancer xenografts following systemic delivery of UPAR or EGFR targeted optical imaging probes}

Next, we wanted to determine if optical imaging probes targeting to tumor cells alone or both tumor and stromal cells have different distributions inside tumors. EGFR-targeted IRDye 800-ScFvEGFR was used as a control imaging probe that only binds to tumor cells but not to stromal cells. After simultaneous delivery of a mixture of Cy5.5-human ATF and IRDye 800-ScFvEGFR into a nude mouse bearing an orthotopic MDA-MB-231 human breast cancer xenograft for 24 hours, whole body optical imaging showed that both optical imaging probes were selectively accumulated in the tumor that enabled tumor imaging (Figure 3). However, strong optical signals were also detected in the liver area of the mouse when the filter set for IRDye 800 was used but not with the filter set for Cy5.5, suggesting a high level of EGFR-targeted imaging probe accumulation in the liver (Figure 3). To determine distribution of the optical signals inside the tumor mass, mice was sacrificed and the intact tumor, without skin and the top thin layer of the tumor surface, was imaged under a high magnification. Using a filter set for Cy5.5 dye, we found strong optical signals both in the center and peripheral of the tumor (Figure 3), suggesting the location of Cy5.5-ATF probes in those tumor regions. However, a strong IRDye 800 signal was detected mostly in the center of the tumor, indicating the accumulation of the EGFR-targeted imaging probes in the center of the tumor but not in the tumor border. Therefore, uPAR-targeted imaging probes may be more suitable for the detection of tumor margins compared with EGFR-targeted probes. 
A. MCF-10DCIS xenograft (H\&E staining)

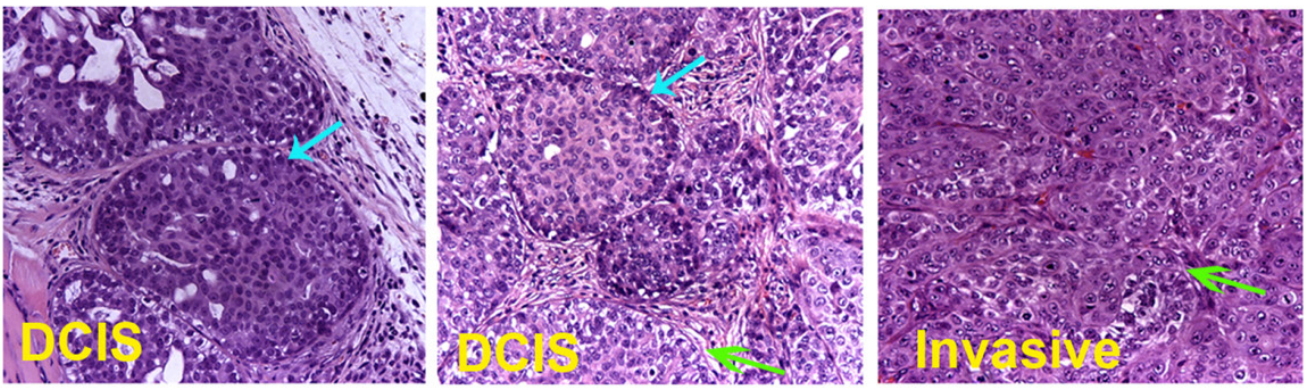

B. MCF-10DCIS xenograft (Immunofluorescence labeling)
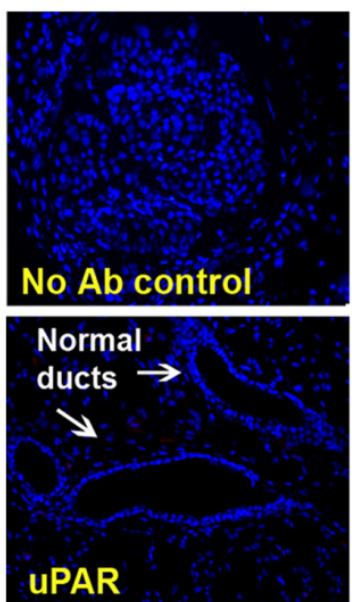
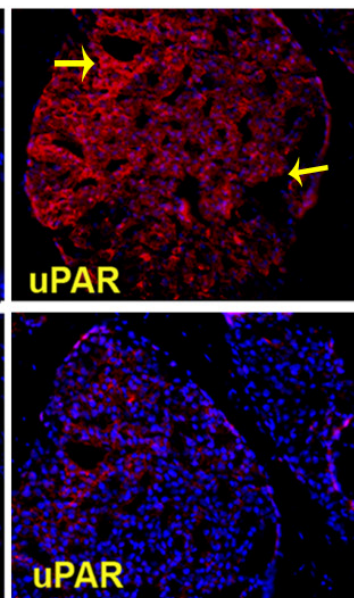
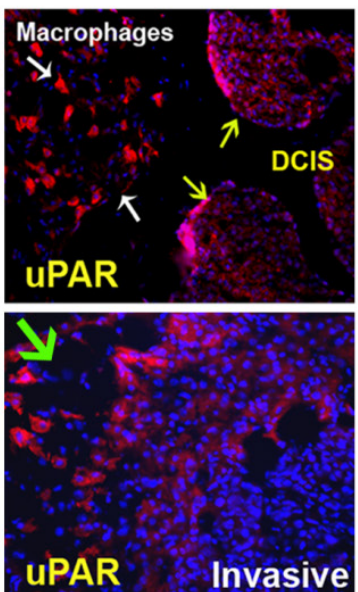

UPAR
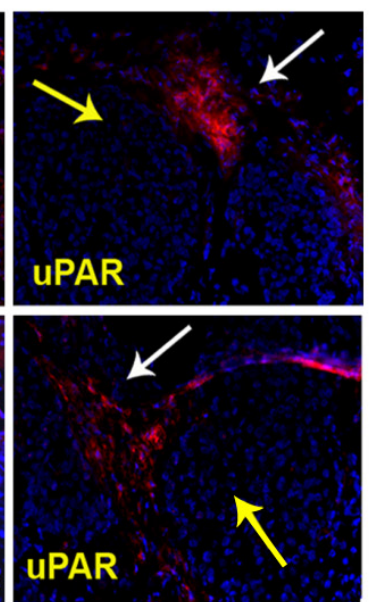

\section{In vivo optical imaging using NIR-830-hATF}

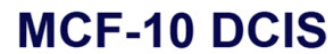

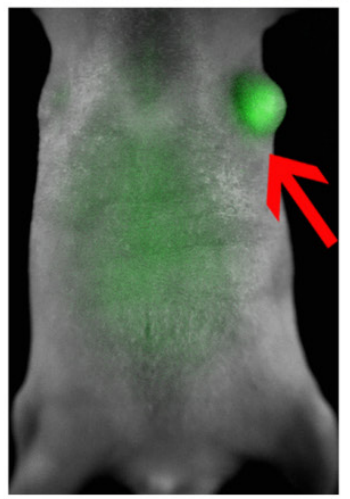

$0.14 x$

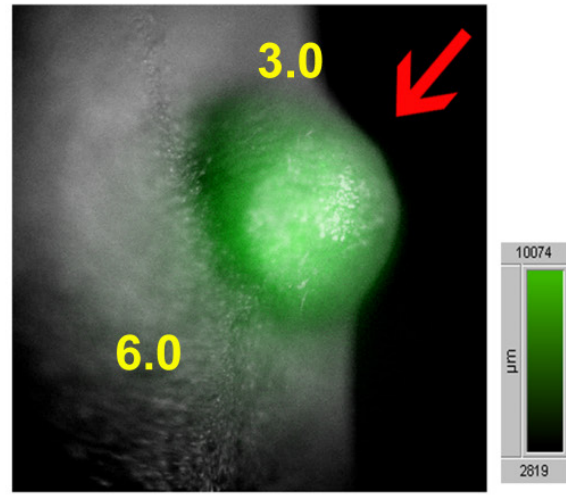

$0.56 x$

Figure 2. Histological characterizations of uPAR expression and non-invasive optical imaging using an uPAR-targeted ATF peptide probe in a human breast cancer xenograft model. A. MCF-IODCIS tumor xenografts. H\&E stained tissue sections showed DCIS characteristics that consisted of solid and comedo types of DCIS (blue arrow) at two weeks, and a mixture of DCIS and invasive lesions (green arrow) at three weeks after tumor cell injection. B. Immunofluorescence labeling. Heterogeneous expression of uPAR was detected in MCF-IODCIS tissues. A high level of uPAR was detected in some DCIS lesions (short yellow arrow) and the invasive edge of the tumor (green arrow). Tumor stroma (white arrows) contained uPAR positive cells, even where the DCIS lacked uPAR expression (long yellow arrows). Blue: Hoechst 33342 background staining. C. Non-invasive in vivo optical imaging of the orthotopic breast cancer xenograft model. A tumor bearing mouse received the tail vein injection of $50 \mu \mathrm{g}$ of NIR-830-human ATF peptides for 24 hours. Optical imaging was performed using the Olympus OV- 100 In Vivo Imaging system at $0.14 \times$ and $0.56 x$ zoom magnifications. Red arrow: an optical signal positive DCIS lesion with defined tumor margin. The ratio of the mean tumor signal to body background near the base of the tumor was 3 . Signal to body background ratio reached 6 when measured in the area away from the tumor base. 


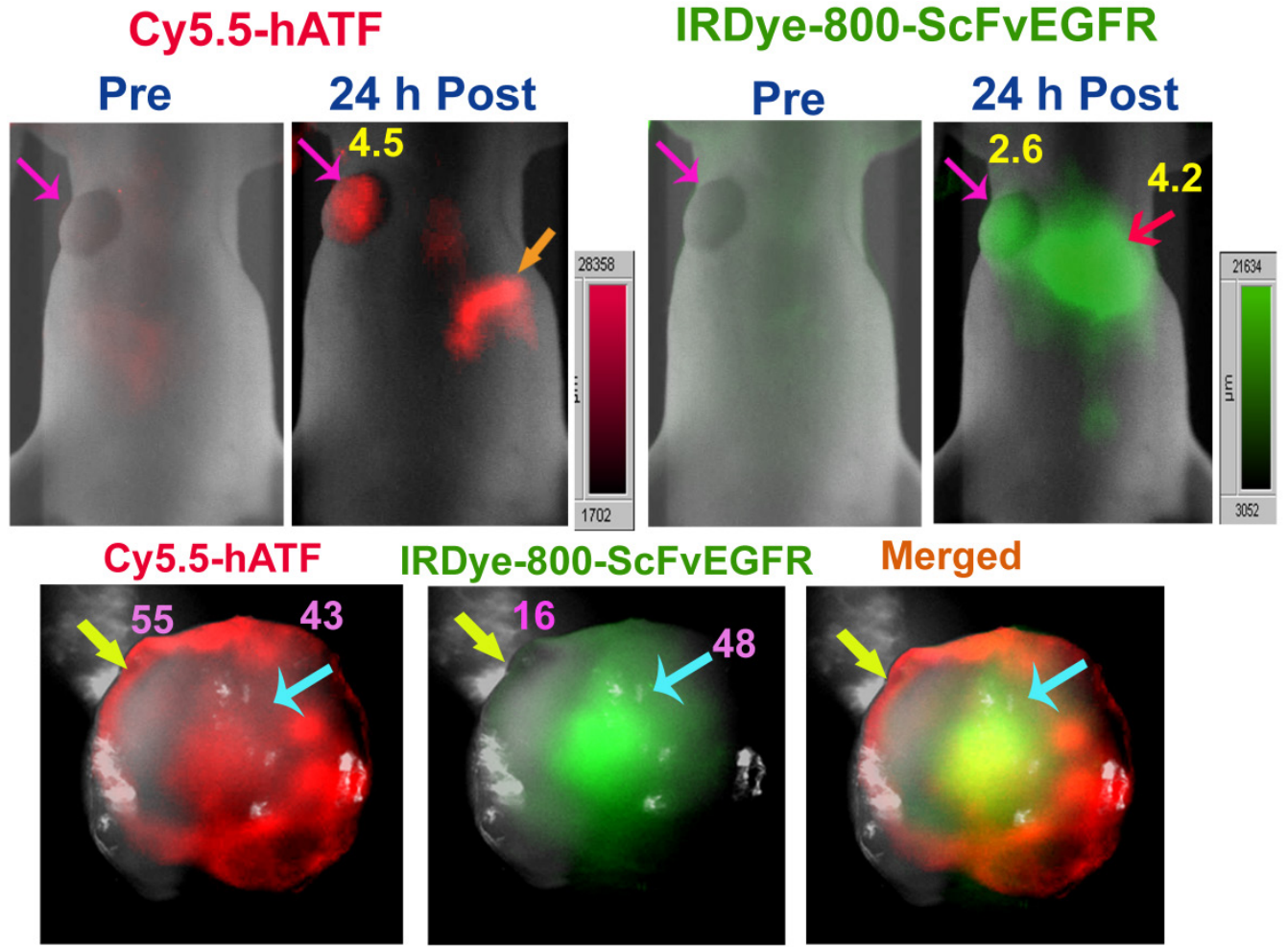

Figure 3. Simultaneous imaging of uPAR and EGFR expression in a human breast cancer xenograft using NIR-dye labeled ATF and ScFvEGFR peptide probes. A nude mouse bearing an orthotopic MDA-MB-23I human breast cancer xenograft received a tail vein delivery of a mixture of $50 \mu g$ of Cy5.5-human ATF and $50 \mathrm{\mu g}$ of IRDye 800-ScFvEGFR for 24 hours. OV- 100 In Vivo Imaging System was used for optical imaging. Strong Cy5.5 and IRdye-800 signals were detected in the tumor xenograft (Upper panel, pink arrows). Cy5.5 signal was also found in the intestine area (Orange arrow). A strong IRDye 800 signal was detected both in the tumor (pink arrow) and liver (red arrow). Numbers shown in the images are signal to body background ratios. A high magnification image of the tumor (zoom, $0.8 \mathrm{x}$ ) showed the presence of Cy5.5-hATF probes (red) in both the tumor center (Lower panel, blue arrows) and the tumor border (Lower panel, yellow arrows). However, a high level of IRDye 800 ScFvEGFR probes (green) was detected in the center of the tumor. Numbers shown in the images are the mean fluorescence intensity of the center or peripheral tumor area.

Figure 4. Species specificity of ATF targeting ligands. Cy5.5-human ATF peptides were injected via the tail vein into the nude mice bearing either MDA-MB-23I human breast cancer xenografts or 4TI mouse mammary tumor in the mammary fat pad. Optical imaging was performed 10 minutes and 24 hours following the imaging contrast administration using the OV-100 In Vivo imaging System with 0.14 and $0.56 x$ zoom magnifications. Strong optical signal was detected in human breast tumor xenografts (yellow arrows) but not in mouse mammary tumors (pink arrows). 24 hour optical image showed a clear tumor border in the mouse bearing the human breast cancer xenograft.

\section{Species specificity of the human ATF imaging probe}

The before-mentioned study used Cy5.5-labeled human ATF that had a high binding affinity to human UPAR [48]. To determine if NIR-dye-labeled human ATF bound to UPAR expressing mouse tumor cells and stromal cells, $50 \mu \mathrm{g}$ of Cy5.5-human ATF peptides were injected via the tail vein in the nude mice bearing either MDA-MB-231 human breast cancer xenografts or $4 \mathrm{~T} 1$ mouse mammary tumors. A strong optical signal was detected in the MDA-MB-231 human breast cancer as early as $10 \mathrm{~min}$ post injection. The optical signal was further enhanced and defined in the tumor

\section{Cy5.5-hATF}
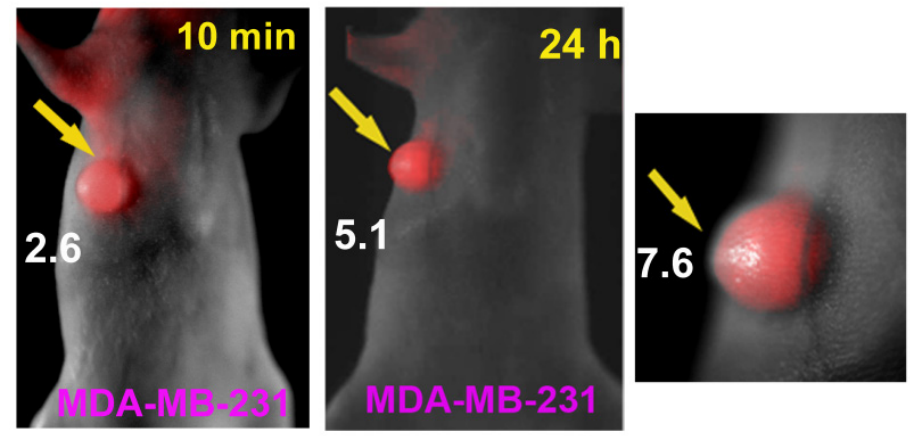

\section{Cy5.5-hATF}
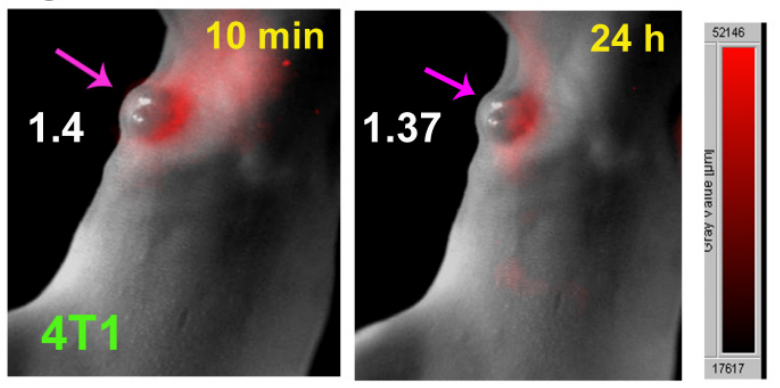

24 hours following the injection (Figure 4). However, in the mice bearing mouse mammary tumors following systemic delivery of human ATF peptide probes, 
the imaging signal was detected in the peripheral base of the tumor $10 \mathrm{~min}$ following injection but was not found in the tumor at 24 hours (Figure 4). Results of this study were consistent with the previous observation that there was species specificity in the binding of the ATF peptide to its cellular receptor [48].

\section{Optical imaging detects drug resistant tumor lesions following systemic delivery of uPAR targeted theranostic nanoparticles in human tumor xenograft models}

One of the major challenges in the effective treatment of human cancer is that a high percentage of cancer patients have already developed locally advanced diseases at diagnosis. Many of them require neoadjuvant therapy before surgery. Our group has developed UPAR-targeted theranostic nanoparticles for image-guided and targeted cancer therapy. To determine whether NIR-dye-labeled and uPAR-targeted theranostic IONPs have the ability to serve as optical imaging probes for the detection of tumor margins and drug resistant residual tumor lesions, we examined the feasibility of optical imaging of orthotopic MCF-10DCIS human breast and MIA PaCa-2 human pancreatic cancer xenografts after the systemic delivery of NIR-830-dye labeled-ATF-IONPs carrying Dox once per week for four treatments. We used a mixture of human and mouse ATF peptides to target both human tumor and mouse stromal cells. 6 days after the last theranostic IONP-Dox delivery, optical imaging revealed strong signals in the MCF-10 DCIS tumor xenograft, which defined the location of the tumor mass (Figure 5A). Prussian blue staining showed scattered blue-IONP positive cells in the necrotic tumor areas, an indication of killing of the tumor cells by ATF-IONP-Dox theranostic nanoparticles (Figure 5A). Furthermore, we detected a high level of the IONP positive cells in the tumor stroma at the center and peripheral tumor areas, where there was an enrichment of tumor vessels (CD31) and tumor macrophages (CD68) (Figure 5A). Therefore, uPAR-targeted theranostic nanoparticles were delivered to both tumor and stromal cells. It seemed that highly proliferative MCF-10DCIS cells were more sensitive to Dox than stromal cells so that there were more IONP positive cells in the tumor stroma following the treatment (Figure 5A).

In the mice bearing human pancreatic cancer xenografts, a strong optical signal was detected in the tumor (Figure 5B), but not in normal organs 6 days after administration of the last treatment of NIR-830-ATF-IONP-Dox nanoparticles. Optical tumor imaging correlated well with the tumor location and size detected by bioluminescence imaging of luciferase positive MIA PaCa-2 tumor cells, and gross ex- amination of the tumor after sacrificing the mice (Figure 5B). In the orthotopic MIA PaCa-2 tumor xenograft, double labeling immunofluorescence of frozen tumor sections showed that CD31 positive tumor vessels were located in the peripheral area adjacent to UPAR expressing tumor cells (Figure 5B). Active tumor stroma immediately adjacent to the tumor border had large numbers of uPAR-positive macrophages (Figure 5B). In the tumor xenograft obtained from the mice that received ATF-IONPs, Prussian blue staining positive cells were found in CD68 positive macrophages and CD68 negative tumor cells (Figure 5B). Prussian blue staining of tumor tissue sections obtained from the mice 6 days following the last treatment of NIR-830-ATF-IONP-Dox revealed a high level of blue IONP containing cells at the tumor edge and tumor-stromal boundary in those drug resistant residual tumors (Figure 5B). Therefore, results of this study suggested that NIR-dye-labeled and uPAR-targeted theranostic nanoparticles are a promising class of multifunctional nanoparticles for targeted therapy followed by identification of tumor margins and small residual tumor lesions for image-guided surgical removal of the tumors.

\section{uPAR-targeted optical tumor imaging in a mouse mammary tumor model}

To determine the optimal time course for tumor imaging, we used an orthotopic 4T1 mouse mammary tumor model and NIR 830 dye labeled mouse ATF peptides, unconjugated or conjugated to IONPs. The use of a mouse targeting ligand in the mouse tumor model should ensure accurate assessment of tumor targeting, intratumoral delivery and distribution of the probes, and the combined effect of targeting both tumor cells and active tumor stroma on tumor imaging. First, $50 \mu \mathrm{g}$ of unconjugated NIR-830-mouse ATF was injected via the tail vein into BALB/c mice bearing 5 to $8 \mathrm{~mm} 4 \mathrm{~T} 1$ mammary tumors. NIR optical imaging was performed at different time points. One day following the injection, optical signal was detected in the tumor and abdominal areas, which were likely from the liver and intestines (Figure 6A). The signal in the tumor was markedly enhanced 2 days following injection. However, optical signals in the intestines were still visible using the OV-100 imaging system, suggesting that NIR-830 dye or NIR-830-dye-peptides were cleared from the liver. Three days following the injection, imaging signals in the tumor reached the highest while the signals in the abdominal cavity became undetectable by non-invasive optical imaging (Figure 6A). We further found that the optical signal persisted in the tumor for over two weeks, although the signal intensity gradually decreased. By two weeks, optical image-guided 
surgical resection was performed. The signal was only detected in the resected mammary tumor but not in the surgical bed of the mice. The tumor tissues were examined by optical imaging and the optical imaging signal was detected within the edge of the resected tissues (Figure 6A). In the mice that received non-targeted NIR-830-MSA probes for 2 days, there was no detectable optical signal in the tumor (Figure $6 \mathrm{~A})$.
Figure 5. uPAR-targeted theranostic IONPs for optical imaging of drug resistant residual tumors. A. Optical imaging of a residual DCIS tumor following uPAR-targeted therapy using NIR-830-ATF-IONP-Dox theranostic nanoparticles. Following four treatments, the residual tumor had a strong optical signal for tumor imaging. CD 31 positive tumor blood vessels and CD 68 positive macrophages were present in the tumor stromal areas (purple arrows) but a few of them were found within the tumor nodules (yellow arrows). Prussian blue staining revealed a low percentage of blue iron positive cells in the necrotic tumor areas (yellow arrow) and a high level of blue iron positive cells in the tumor stroma (blue arrow). B. Detection of drug resistant residual pancreatic cancers by optical imaging after the treatment of an orthotopic human pancreatic cancer xenograft model with NIR-830-ATF-IONP-Dox. Optical imaging was done 6 days following the last nanoparticle injection. Strong optical signal was detected in the tumor, which was confirmed by bioluminescence imaging and gross examination of the tumor location after sacrificing the mouse. Double labeling fluorescence using CD3I (green) and uPAR (red) antibodies showed tumor vessels in the peripheral area near the tumor edge where tumor cells expressed a high level of uPAR (yellow arrow). CD68 positive macrophages were found adjacent to uPAR-expressing tumor border (red) and most tumor associated macrophages (green) also expressed uPAR (yellow or orange). Prussian blue positive cells were found in CD68 expressing macrophages (red arrow) and in tumor cells. Furthermore, high levels of Prussian blue positive cells (yellow arrows) were detected in the invasive edge of the tumor but not in nearby normal pancreatic tissues (green arrows). Red background staining is nuclear fast red.

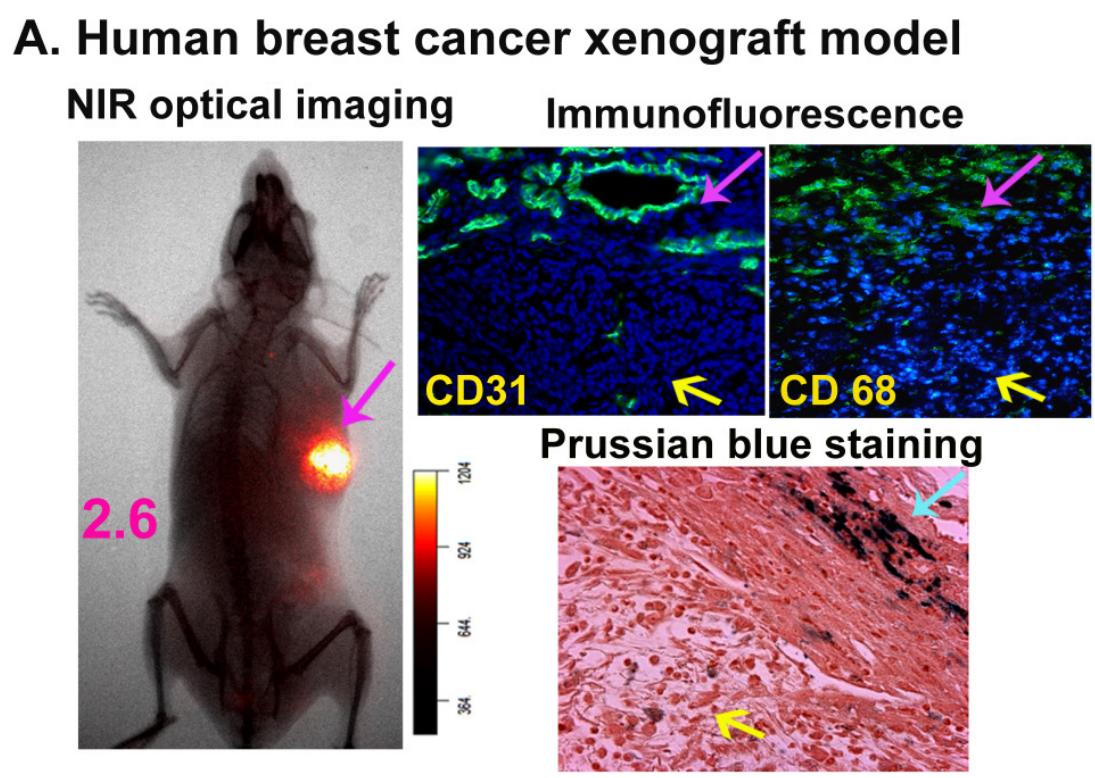

B. Human pancreatic cancer xenograft model 2.2

Histological analysis
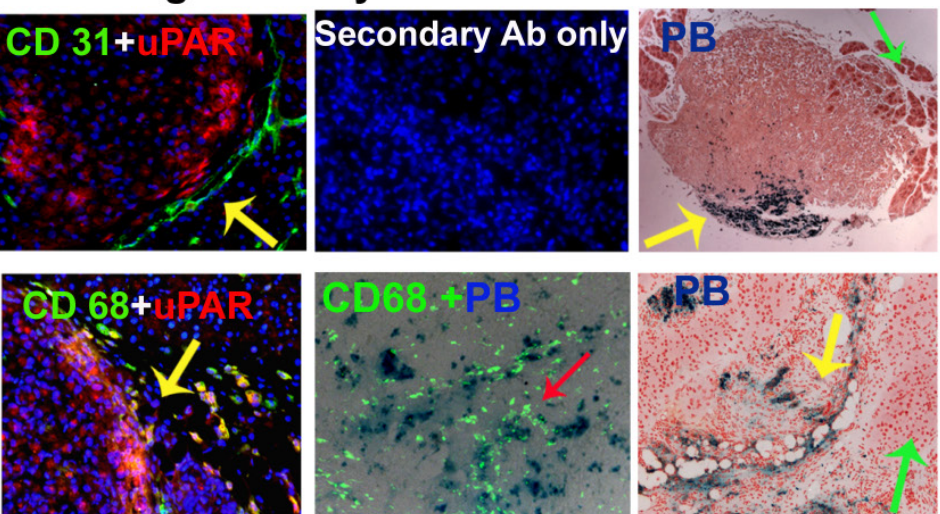
A. NIR-830-mATF 0.82
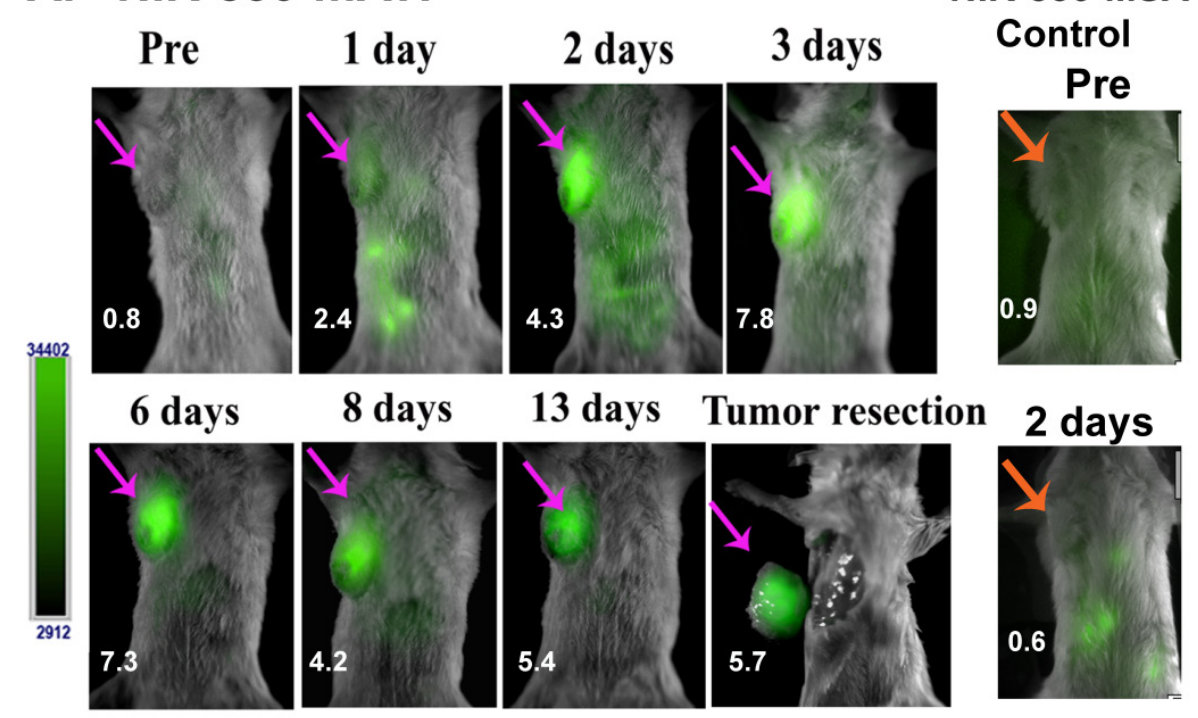

NIR-830-MSA

\section{B. NIR-830-mATF-IONP}
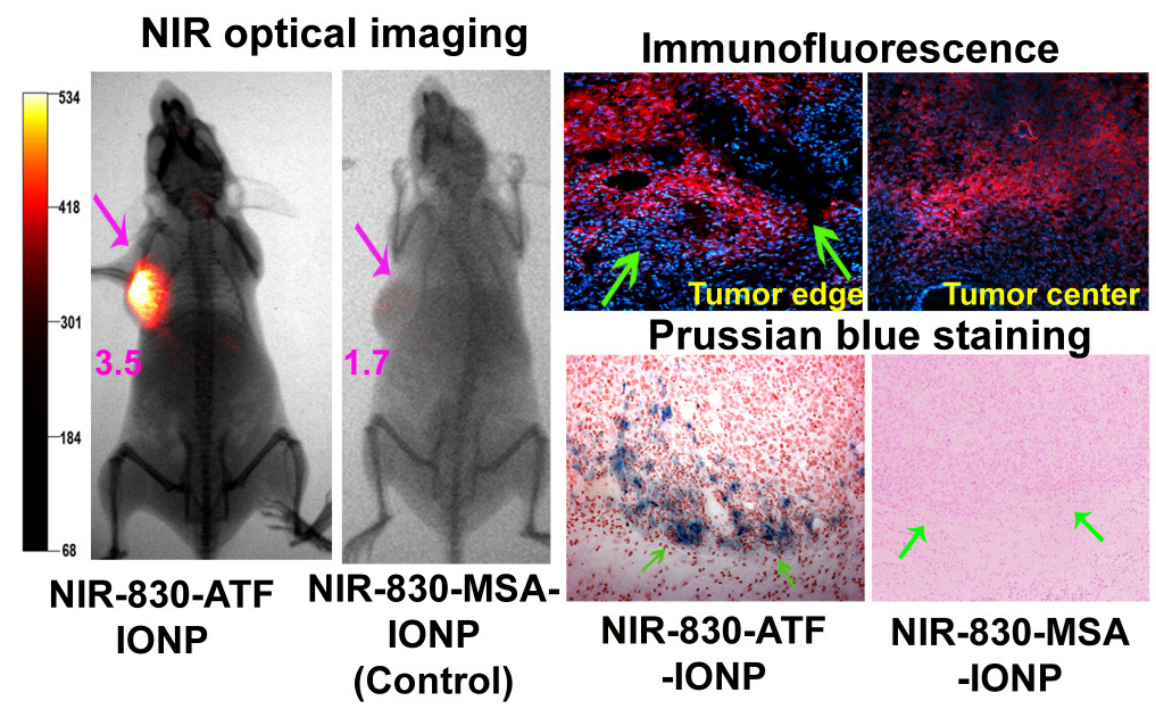

Figure 6. Time course optical imaging of mouse mammary tumors using NIR-830-mouse ATF imaging probes. A. A mouse bearing an orthotopic 4TI mouse mammary tumor received tail vein delivery of $50 \mu \mathrm{g}$ of NIR-830-mouse ATF peptide probes. Optical imaging was performed different time points. Strong optical signal was detected 2 to 3 days following the probe delivery and was still detectable at I 3 days. Numbers in the images show the ratio of the mean fluorescence intensity of the tumor and body background. The tumor was then removed by image-guided surgery at 13 days. The mean fluorescence intensity of the tumor area is 2.1 fold higher than that of the resected surrounding normal tissues. The mean fluorescence intensity of the tumor is 4.3 fold higher than the signal of the surgical cavity. The mean fluorescence intensity in the surgical bed is about the same as the body background (ratio: 0.92). Similar results were observed in three individual mice. B. Optical imaging of a mouse bearing 4TI mouse mammary tumor after receiving 100 picomolar of NIR-830-mouse ATF-IONP for 48 hours. Specificity of uPAR-targeted tumor imaging was demonstrated by comparing the ratios of the mean fluorescence signals of the tumor and body background obtained from the mouse that received NIR-830-mouse ATF-IONP (3.46) with that of non-targeted NIR-830-BSA-IONP injected mouse (I.67). Histological analysis using immunofluorescence labeling detected uPAR expression in the tumor with a high level in the invasive tumor edge (green arrows). Prussian blue staining showed that a high level of IONPs also accumulated in the edge of the tumor obtained from NIR-830-ATF-IONP injected mice but not in tumor tissues from NIR-830-MSA-IONP injected mice.

Next, we examined the imaging specificity and intratumoral distribution of mouse ATF-conjugated IONPs in the mammary tumor model. 100 pmol of the NIR-830 dye-mouse ATF-IONPs were injected into BALB/c mice bearing 4T1 mammary tumors. Three days after the nanoparticle delivery, whole body optical imaging showed a strong NIR signal in the mammary tumor but not in any other areas of the body. The optical tumor image well delineated the tumor margin of the mammary tumor. Histological analysis of the 4T1 tumor tissue sections showed that UPAR was expressed in the tumor with a high level in the invasive edge and tumor border areas (Figure 6B). Prussian blue staining revealed a high level of NIR-830-ATF-IONPs in the tumor border area but not in the normal mammary tissue (Figure 6B). On the 
other hand, the tumor bearing mice that received non-targeted NIR-830 MSA-IONP lacked the optical signal in the tumor and Prussian blue positive cells were not found in the frozen tumor sections (Figure $6 B)$.

\section{Discussion}

Effective surgery by complete removal of tumors holds great promise to reduce the incidences of local tumor recurrence due to the presence of residual tumors in and near the surgical cavity. The development of novel intraoperative approaches for fast and accurate identification of tumor lesions should have the potential to significantly improve survival of cancer patients $[5,6,13,49]$. Optical imaging in combination with targeted imaging contrast agents provides a fast and simple means to detect tumors with high sensitivity and specificity [50]. At present, various types of optical contrast agents have been developed and their effects on the detection of tumors by non-invasive and intraoperative imaging have been demonstrated in animal tumor models and in human patients [16, 18, 19, 22, 27]. Multimodality imaging probes with the capability for optical imaging and MRI, or optical imaging and positron emission tomography (PET) have been developed and the feasibility for tumor imaging using different imaging modalities have also been demonstrated in animal tumor models $[20,51,52]$. Although increasing evidence shows that cell surface receptor-targeted imaging probes are able to enhance specificity and sensitivity of tumor imaging, it is unclear whether there are differences in the intensity and location of optical signals among the imaging probes targeted to different cell types in the tumor.

A major limitation of optical imaging is its low sensitivity in the detection of optical probes located deep inside the tumor $[16,50]$. Most excitation sources have a high efficiency in exciting imaging probes located several millimeters to a centimeter deep in the tumor $[21,22,50]$. For a human tumor that is larger than 2 centimeters, it is important for the optical imaging probes to accumulate at a high concentration in the peripheral areas of the tumor so that they can be efficiently excited and emit without extensive attenuations in the tissues. Therefore, it is likely that imaging probes that are selectively targeted to the invasive tumor edge and active tumor stroma have the potential for the development of image-guided surgery. In this study, we evaluated the ability of uPAR targeted optical imaging probes, either as unconjugated NIR-dye-ATF peptide or NIR-830-ATF-IONP nanoparticle probes, in optical imaging of tumors in orthotopic human breast and pancreatic cancer xenograft models and a mouse mammary tumor model.
Results of our study showed that UPAR is expressed in the tumor and stromal cells in those animal tumor models. Systemic delivery of uPAR-targeted imaging probes or theranostic nanoparticles led to the accumulation of the nanoparticles in the tumor with a higher level in the tumor border, which allowed delineation of the tumor margin by optical imaging. Optical imaging properties and the unique intratumoral distribution of uPAR-targeted imaging probes support the potential of further development of uPAR-targeted optical imaging contrast agents for the detection of tumor margins.

Breaking of the extracellular matrix by activated proteases promotes upregulation of uPAR in aggressive and invasive tumor cell populations that are likely located in the invasive tumor edge, where there are extensive angiogenesis and active tumor stroma. One of the limitations in intratumoral delivery of imaging and therapeutic agents is low delivery efficiency, especially in the central hypoxic tumor regions. An imaging contrast targeting a biomarker that is highly expressed in the tumor boundary has the opportunity to interact with its cell surface target without relying on efficient intratumoral delivery and distribution. This fits well with the clinical need of the tumor border detection. Since the majority of cell-surface targets are not tumor cell specific, the imaging contrast effect mainly depends upon the differential levels of the receptor expression in the normal, tumor, and stromal cells. For example, human breast cancer cells have 13,000 to 50,000 uPAR per cell while primary normal human mammary epithelial cells only have 2,500 uPAR per cell [53]. Recent studies have shown that the interaction of uPA with uPAR leads to the internalization of the ligand/receptor complex, which may contribute to enhanced tumor retention of the uPAR targeted probes [54].

The importance of the uPA/uPAR system in tumor matastasis and angiogenesis provides a strong rationale for research efforts in search of potent inhibitors for this system. Recombinant ATF and small peptide mimetics of the receptor binding domain of uPA have been used as uPAR inhibitors and showed tumor inhibitory effects in animal tumor models [54-57]. Although the clinical potential for cancer treatment using ATF or small peptide mimetics alone or in combination with other therapeutic approaches has yet to be determined, those ligands are good candidates for the development of uPAR-targeted imaging and therapeutic agents since it is possible to produce nanomolar affinity and stable targeting ligands in large quantity for translational applications. Although anti-uPAR or uPA antibodies have been used as targeting ligands for the development of uPAR targeted imaging and therapeutic 
agents $[40,58]$, they have a molecular weight of 150 $\mathrm{KDa}$, which is 9 to 100 fold larger than ATF peptides or peptide mimetics, respectively. Furthermore, antibodies have a long blood half life ( 72 hours), which may increase body background [59]. A 9-mer linear peptide mimetic (AE105) with a tight binding affinity to human UPAR has been shown to be a strong antagonist of the uPA/uPAR interaction. Systemic delivery of ${ }^{64} \mathrm{Cu}$-labeled AE105-DOTA showed target specificity in UPAR expressing U87MG human glioblastoma xenografts and the ability of PET imaging of the tumor [60]. However, blood clearance time of small peptide mimetics is relatively short, which may limit the accumulation of sufficient amounts of the targeted imaging probes for sensitive detection of tumor margins.

One of the key issues to be considered for the preclinical development of the ATF peptides as UPAR targeted agents is species specificity. For example, human ATF has a high affinity to human uPAR $\left(K_{D}=\right.$ $\left.2.4 \times 10^{-10} \mathrm{M}\right)$ but a low affinity to mouse uPAR $\left(\mathrm{K}_{\mathrm{D}}=6.8 \times 10^{-8} \mathrm{M}\right)$ [48]. On the other hand, mouse ATF has a high affinity to mouse $\mathrm{UPAR}\left(\mathrm{K}_{\mathrm{D}}=1.7 \times 10^{-10} \mathrm{M}\right)$. However, it could bind to human uPAR with an intermediate affinity $\left(1.9 \times 10^{-8} \mathrm{M}\right)$ [48]. Consistent with the results of previous studies, we observed strong imaging signals in the human breast cancer xenografts after systemic delivery of human ATF peptides. However, human ATF imaging probes were not able to target to mouse mammary tumors. Therefore, differential binding affinity of ATF peptides derived from different species should be taken into consideration when designing preclinical targeted imaging and biodistribution studies. As shown in Figure 5, for human tumor xenograft models in nude mice, a mixture of human and mouse ATF can be used to determine the targeted imaging and therapeutic effects in both tumor and stromal cells.

A major challenge in identifying tumor margin and small residual tumor lesions in breast tissues following neoadjuvant therapy is the presence of scar-like fibrosis, wound-healing, and chronic inflammation in the tumor bed that makes it difficult to visualize the tumor mass and margins. Results of our study suggest that uPAR-targeted NIR-dyetheranostic nanoparticles have the potential to serve as multifunctional agents for targeted therapy and image-guided surgery. NIR optical imaging has a high sensitivity to detect residual resistant tumors with only a small percentage of tumor cells containing theranostic nanoparticles. Our results also showed that targeting theranostic nanoparticles to tumor stromal cells that are relatively resistant to Dox due to a lower cell proliferation rate than tumor cells, al- lowed for the detection of the residual tumor by optical imaging.

Although optical imaging systems used in this study could not be applied directly to image-guided surgery in human patients, we are in the process of evaluating several hand-held optical imaging systems, including spectroscopic, fluorescence molecular tomography, and photoacoustic imaging devices, to determine the effects of image-guided surgery on tumor recurrences and long-term survival in several animal tumor models.

\section{Acknowledgments}

We thank Dr. Fred R. Miller at Wayne State University for providing 4T1 cell line, Dr. Gregory Adams at Fox Chase Cancer Center for single chain EGFR antibody plasmid construct ( $\mathrm{ScFv}$ B10), Dr. Xinjian Zhang for constructing mouse ATF expression construct, Dr. Rosa Hwang for luciferase stable MIA PaCa-2 cell line, Dr. Hongyu Zhou for uPAR staining of 4T1 tumor tissue sections, and Dr. Erica Bozeman for editing the manuscript. We would also like to thank the Integrated Cellular Imaging Core for their assistance on the OV-100 In Vivo imaging system. This research project was supported by $\mathrm{NIH} / \mathrm{NCI}$ grants, R01CA133722 (Yang), R01 CA154129A01 (Yang), and U54 CA119338 (Nie), and the Nancy Panoz Endowed Chair Funds (Yang).

\section{Competing Interests}

The authors have declared that no competing interest exists.

\section{References}

1. Balch GC, Mithani SK, Simpson JF, Kelley MC. Accuracy of intraoperative gross examination of surgical margin status in women undergoing partial mastectomy for breast malignancy. Am Surg. 2005;71(1): 22-7.

2. Abraham SC, Fox K, Fraker D, Solin L, Reynolds C. Sampling of grossly benign breast reexcisions: a multidisciplinary approach to assessing adequacy. Am J Surg Pathol. 1999;23(3): 316-22.

3. Sigal-Zafrani B, Lewis JS, Clough KB, et al. Histological margin assessment for breast ductal carcinoma in situ: precision and implications. Mod Pathol. 2004;17(1): 81-8.

4. Verbeke CS, Menon KV. Redefining resection margin status in pancreatic cancer. HPB. (Oxford) 2009;11(4): 282-9.

5. Howard TJ, Krug JE, Yu J, et al. A margin-negative R0 resection accomplished with minimal postoperative complications is the surgeon's contribution to long-term survival in pancreatic cancer. J Gastrointest Surg. 2006;10(10): $1338-45$

6. Lee J, Lee S, Bae Y. Multiple margin positivity of frozen section is an independent risk factor for local recurrence in breast-conserving surgery. J Breast Cancer. 2012;15(4): 420-6

7. Jung W, Kang E, Kim SM, et al. Factors Associated with Re-excision after Breast-Conserving Surgery for Early-Stage Breast Cancer. J Breast Cancer. 2012;15(4): 412-9.

8. Chang DK, Johns AL, Merrett ND, et al. Margin clearance and outcome in resected pancreatic cancer. J Clin Oncol. 2009;27(17): 2855-62.

9. Kimbrough CW, St Hill CR, Martin RC, McMasters KM, Scoggins CR. Tumor-positive resection margins reflect an aggressive tumor biology in pancreatic cancer. J Surg Oncol. 2013;107(6): 602-7.

10. Neoptolemos JP, Stocken DD, Dunn JA, et al. Influence of resection margins on survival for patients with pancreatic cancer treated by adjuvant chemoradiation and/or chemotherapy in the ESPAC-1 randomized controlled trial. Ann Surg. 2001;234(6): 758-68.

11. Konstantinidis IT, Warshaw AL, Allen JN, et al. Pancreatic ductal adenocarcinoma: is there a survival difference for R1 resections versus locally 
advanced unresectable tumors? What is a "true" R0 resection? Ann Surg. 2013;257(4): 731-6.

12. Assersohn L, Powles TJ, Ashley S, et al. Local relapse in primary breast cancer patients with unexcised positive surgical margins after lumpectomy, radiotherapy and chemoendocrine therapy. Ann Oncol. 1999;10(12): 1451-5.

13. Bolger JC, Solon JG, Power C, Hill AD. Analysis of margin index as a method for predicting residual disease after breast-conserving surgery in a European cancer center. Ann Surg Oncol. 2012;19(1): 207-11.

14. Esposito I, Kleeff J, Bergmann F, et al. Most pancreatic cancer resections are R1 resections. Ann Surg Oncol. 2008;15(6): 1651-60.

15. Sahoo S, Lester SC. Pathology of breast carcinomas after neoadjuvant chemotherapy: an overview with recommendations on specimen processing and reporting. Arch Pathol Lab Med. 2009;133(4): 633-42.

16. Weissleder R, Tung $\mathrm{CH}$, Mahmood U, Bogdanov A, Jr. In vivo imaging of tumors with protease-activated near-infrared fluorescent probes. Nat Biotechnol. 1999;17(4): 375-8.

17. Becker A, Hessenius C, Licha K, et al. Receptor-targeted optical imaging of tumors with near-infrared fluorescent ligands. Nat Biotechnol. 2001;19(4): 327-31.

18. Ye Y, Bloch S, Xu B, Achilefu S. Design, synthesis, and evaluation of near infrared fluorescent multimeric RGD peptides for targeting tumors. J Med Chem. 2006;49(7): 2268-75.

19. van Dam GM, Themelis G, Crane LM, et al. Intraoperative tumor-specific fluorescence imaging in ovarian cancer by folate receptor-alpha targeting: first in-human results. Nat Med. 2011;17(10): 1315-9.

20. Bradbury MS, Phillips E, Montero PH, et al. Clinically-translated silica nanoparticles as dual-modality cancer-targeted probes for image-guided surgery and interventions. Integr Biol (Camb). 2013;5(1): 74-86.

21. Keereweer S, Van Driel PB, Snoeks TJ, et al. Optical image-guided cancer surgery: challenges and limitations. Clin Cancer Res. 2013;19(14): 3745-54.

22. Vahrmeijer AL, Hutteman M, van der Vorst JR, van de Velde CJ, Frangioni JV. Image-guided cancer surgery using near-infrared fluorescence. Nat Rev Clin Oncol. 2013; Epub ahead of print. Doi: 10.1038/nrclinonc.2013.123.

23. Yang L, Mao H, Cao Z, et al. Molecular imaging of pancreatic cancer in an animal model using targeted multifunctional nanoparticles. Gastroenterology. 2009;136(5): 1514-25

24. Nguyen QT, Olson ES, Aguilera TA, et al. Surgery with molecular fluorescence imaging using activatable cell-penetrating peptides decreases residual cancer and improves survival. Proc Natl Acad Sci U S A. 2010;107(9): $4317-22$.

25. Kelly K, Alencar H, Funovics M, Mahmood U, Weissleder R. Detection of invasive colon cancer using a novel, targeted, library-derived fluorescent peptide. Cancer Res. 2004;64(17): 6247-51.

26. Ke S, Wen X, Gurfinkel M, et al. Near-infrared optical imaging of epidermal growth factor receptor in breast cancer xenografts. Cancer Res. 2003;63(22): 7870-5.

27. Yang L, Peng $\mathrm{XH}$, Wang $\mathrm{YA}$, et al. Receptor-targeted nanoparticles for in vivo imaging of breast cancer. Clin Cancer Res. 2009;15(14): 4722-32.

28. Yang L, Mao H, Wang YA, et al. Single chain epidermal growth factor receptor antibody conjugated nanoparticles for in vivo tumor targeting and imaging. Small. 2009;5(2): 235-43.

29. Cai W, Shin DW, Chen K, et al. Peptide-labeled near-infrared quantum dots for imaging tumor vasculature in living subjects. Nano Lett. 2006;6(4): 669-76.

30. Moore A, Medarova Z, Potthast A, Dai G. In vivo targeting of underglycosylated MUC-1 tumor antigen using a multimodal imaging probe. Cancer Res. 2004;64(5): 1821-7.

31. Blasi F, Carmeliet P. uPAR: a versatile signalling orchestrator. Nat Rev Mol Cell Biol. 2002;3(12): 932-43.

32. Hemsen A, Riethdorf L, Brunner $\mathrm{N}$, et al. Comparative evaluation of urokinase-type plasminogen activator receptor expression in primary breast carcinomas and on metastatic tumor cells. Int J Cancer. 2003;107(6): 903-9.

33. He $Y$, Liu $X D$, Chen $Z Y$, et al. Interaction between cancer cells and stromal fibroblasts is required for activation of the uPAR-uPA-MMP-2 cascade in pancreatic cancer metastasis. Clin Cancer Res. 2007;13(11): 3115-24.

34. Kjaergaard M, Hansen LV, Jacobsen B, Gardsvoll H, Ploug M. Structure and ligand interactions of the urokinase receptor (uPAR). Front Biosci. 2008;13: 5441-61.

35. Cantero D, Friess H, Deflorin J, et al. Enhanced expression of urokinase plasminogen activator and its receptor in pancreatic carcinoma. Br J Cancer. 1997;75(3): 388-95

36. Solberg H, Ploug M, Hoyer-Hansen G, Nielsen BS, Lund LR. The murine receptor for urokinase-type plasminogen activator is primarily expressed in tissues actively undergoing remodeling. J Histochem Cytochem. 2001;49(2): 237-46.

37. Salden M, Splinter TA, Peters HA, et al. The urokinase-type plasminogen activator system in resected non-small-cell lung cancer. Rotterdam Oncology Thoracic Study Group. Annals of oncology : official journal of the European Society for Medical Oncology / ESMO. 2000;11(3): 327-32.

38. Kotzsch $\mathrm{M}$, Bernt $\mathrm{K}$, Friedrich $\mathrm{K}$, et al. Prognostic relevance of tumour cell-associated uPAR expression in invasive ductal breast carcinoma. Histopathology. 2010;57(3): 461-71.

39. Nielsen BS, Rank F, Illemann M, Lund LR, Dano K. Stromal cells associated with early invasive foci in human mammary ductal carcinoma in situ coexpress urokinase and urokinase receptor. International journal of cancer. Journal international du cancer. 2007;120(10): 2086-95.
40. LeBeau AM, Duriseti S, Murphy ST, et al. Targeting uPAR with antagonistic recombinant human antibodies in aggressive breast cancer. Cancer Res. 2013;73(7): 2070-81.

41. Chen $\mathrm{Y}$, Zheng B, Robbins DH, et al. Accurate discrimination of pancreatic ductal adenocarcinoma and chronic pancreatitis using multimarker expression data and samples obtained by minimally invasive fine needle aspiration. Int J Cancer. 2007;120(7): 1511-7.

42. Xue A, Scarlett CJ, Jackson CJ, Allen BJ, Smith RC. Prognostic significance of growth factors and the urokinase-type plasminogen activator system in pancreatic ductal adenocarcinoma. Pancreas. 2008;36(2): 160-7.

43. Pyke C, Graem N, Ralfkiaer E, et al. Receptor for urokinase is present in tumor-associated macrophages in ductal breast carcinoma. Cancer Res. 1993;53(8): 1911-5.

44. Hildenbrand R, Schaaf A. The urokinase-system in tumor tissue stroma of the breast and breast cancer cell invasion. Int J Oncol. 2009;34(1): 15-23.

45. Zhao Q, Jiang H, Cao Z, Yang L, Mao H, Lipowska M. A handheld fluorescence molecular tomography system for intraoperative optical imaging of tumor margins. Med Phys. 2011;38(11): 5873-8.

46. Zhou Z, Chen $\mathrm{H}$, Lipowska $\mathrm{M}$, et al. A dual-modal magnetic nanoparticle probe for preoperative and intraoperative mapping of sentinel lymph nodes by magnetic resonance and near infrared fluorescence imaging. J Biomater Appl. 2013;28(1): 100-11.

47. Miller FR, Santner SJ, Tait L, Dawson PJ. MCF10DCIS.com xenograft model of human comedo ductal carcinoma in situ. J Natl Cancer Inst. 2000;92(14): 1185-6.

48. Lin L, Gardsvoll H, Huai Q, Huang M, Ploug M. Structure-based engineering of species selectivity in the interaction between urokinase and its receptor: implication for preclinical cancer therapy. J Biol Chem. 2010;285(14): 10982-92.

49. Spivack B, Khanna MM, Tafra L, Juillard G, Giuliano AE. Margin status and local recurrence after breast-conserving surgery. Arch Surg. 1994;129(9): 952-6; discussion 56-7.

50. Weissleder R. Molecular imaging in cancer. Science. 2006;312(5777): 1168-71.

51. Holland JP, Normand G, Ruggiero A, Lewis JS, Grimm J. Intraoperative imaging of positron emission tomographic radiotracers using Cerenkov luminescence emissions. Mol Imaging. 2011;10(3): 177-86.

52. Ploug M. Structure-Driven Design of Radionuclide Tracers for Non-Invasive Imaging of uPAR and Targeted Radiotherapy. The Tale of a Synthetic Peptide Antagonist. Theranostics. 2013;3(7): 467-76.

53. Li Y, Wood N, Yellowlees D, Donnelly PK. Cell surface expression of urokinase receptor in normal mammary epithelial cells and breast cancer cell lines. Anticancer Res. 1999;19(2A): 1223-8.

54. Liu S, Bugge TH, Leppla SH. Targeting of tumor cells by cell surface urokinase plasminogen activator-dependent anthrax toxin. J Biol Chem. 2001;276(21): $17976-84$.

55. de Virgilio M, Silvestris F. Urokinase receptor (uPAR) ligand based recombinant toxins for human cancer therapy. Curr Pharm Des. 2011;17(19): 1979-83.

56. Ploug M, Ostergaard S, Gardsvoll H, et al. Peptide-derived antagonists of the urokinase receptor. affinity maturation by combinatorial chemistry, identification of functional epitopes, and inhibitory effect on cancer cell intravasation. Biochemistry. 2001;40(40): 12157-68.

57. Berkenblit A, Matulonis UA, Kroener JF, et al. A6, a urokinase plasminogen activator (uPA)-derived peptide in patients with advanced gynecologic cancer: a phase I trial. Gynecologic oncology. 2005;99(1): 50-7.

58. O'Halloran TV, Ahn R, Hankins P, Swindell E, Mazar AP. The Many Spaces of uPAR: Delivery of Theranostic Agents and Nanobins to Multiple Tumor Compartments through a Single Target. Theranostics. 2013;3(7): 496-506.

59. Rosenblum MG, Kavanagh JJ, Burke TW, et al. Clinical pharmacology, metabolism, and tissue distribution of 90Y-labeled monoclonal antibody B72.3 after intraperitoneal administration. J Natl Cancer Inst. 1991;83(22): 1629-36.

60. Li ZB, Niu G, Wang H, et al. Imaging of urokinase-type plasminogen activator receptor expression using a $64 \mathrm{Cu}$-labeled linear peptide antagonist by microPET. Clin Cancer Res. 2008;14(15): 4758-66. 